\title{
Traumatic cerebrovascular injury: clinical characteristics and illustrative cases
}

\author{
Thara Tunthanathip, MD, Nakornchai Phuenpathom, MD, Sakchai Sae-Heng, MD, \\ Thakul Oearsakul, MD, Ittichai Sakarunchai, MD, and Anukoon Kaewborisutsakul, MD
}

Neurosurgical Unit, Department of Surgery, Faculty of Medicine, Prince of Songkla University, Hat Yai, Songkhla, Thailand

OBJECTIVE Traumatic cerebrovascular injury (TCVI) is a rare and serious complication of traumatic brain injury (TBI). Various forms of TCVIs have been reported, including occlusions, arteriovenous fistulas, pseudoaneurysms, and transections. They can present at a variety of intervals after TBI and may manifest as sudden episodes, progressive symptoms, and even delayed fatal events. The purpose of this study was to analyze cases of TCVI identified at a single institution and further explore types and characteristics of these complications of TBI in order to improve recognition and treatment of these injuries.

METHODS The authors performed a retrospective review of cases of TCVI identified at their institution between 2013 and 2016. A total of 5178 patients presented with TBI during this time period, and 42 of these patients qualified for a diagnosis of TCVI and had adequate medical and imaging records for analysis. Data from their cases were analyzed, and 3 illustrative cases are presented in detail.

RESULTS The most common type of TCVI was arteriovenous fistula (86.4\%), followed by pseudoaneurysm (11.9\%), occlusion (2.4\%), and transection (2.4\%). The mortality rate of patients with TCVI was $7.1 \%$.

CONCLUSIONS The authors describe the clinical characteristics of patients with TCVI and provide data from a series of 42 cases. It is important to recognize TCVI in order to facilitate early diagnosis and treatment.

https://thejns.org/doi/abs/10.3171/2019.8.FOCUS19382

KEYWORDS traumatic cerebrovascular injury; vascular injury; traumatic brain injury; penetrating brain injury

$\mathrm{T}$ RAUMATIC cerebrovascular injury (TCVI) is a cerebrovascular structural injury caused by a traumatic brain injury (TBI). ${ }^{14}$ TCVI is an uncommon complication of TBI and has been reported to occur in $0.8 \%-$ $1.7 \%$ of cases. ${ }^{4,22,27,28}$ TCVIs have been reported in various forms, including occlusion from vasospasm, dissection, or thromboembolism; arteriovenous fistula (AVF); pseudoaneurysm; and transection of a vessel., ${ }^{3,4}$ The timing of TCVI with respect to the causal TBI may be early or late. ${ }^{22}$ Additionally, the clinical presentation of TCVI varies according to the pathology. Sudden onset of neurological deficits from cerebrovascular occlusions tends to occur in acute periods, ${ }^{11}$ whereas signs and symptoms of AVFs are ongoing and progressive for several weeks after the initial injury. ${ }^{16}$ Pseudoaneurysms have been reported in various natural histories. Larson et al. reviewed cases of traumatic intracranial aneurysms and described the presentation as ranging from asymptomatic incidental findings to fatal massive epistaxis. ${ }^{15}$

The objective of the present study was to describe the clinical characteristics of patients with TCVI. Toward that end, we present both descriptive analyses of a series of cases identified at our institution and details of 3 illustrative cases from that series.

\section{Methods \\ Population Study}

This study was a retrospective review of cases involving patients treated at Songklanagarind Hospital, a level I trauma center, between 2013 and 2016 with a diagnosis of TBI. The patients' medical records and neuroimaging studies were obtained from the hospital information system.

According to the clinical practice at our institute, TBI patients who underwent CT angiography (CTA) or cerebral angiography or both during the acute period had one or more of the following: neurological deficits that could not be clarified from findings on CT of the brain; a Glasgow Coma Scale (GCS) score of 3-8; thick, diffuse subarachnoid hemorrhage; severe, localized subarachnoid

ABBREVIATIONS AVF = arteriovenous fistula; CTA = CT angiography; GCS = Glasgow Coma Scale; GOS = Glasgow Outcome Scale; ICA = internal carotid artery; MCA = middle cerebral artery; $\mathrm{PCA}=$ posterior cerebral artery; $\mathrm{PCOA}=$ posterior communicating artery; $\mathrm{TBI}=$ traumatic brain injury; $\mathrm{TCVI}=$ traumatic cerebrovascular injury. SUBMITTED May 18, 2019. ACCEPTED August 16, 2019. INCLUDE WHEN CITING DOI: 10.3171/2019.8.FOCUS19382. 
hemorrhage; excessive bleeding from the mouth, nose, or ear(s); cervical spine fractures at the C1-3 levels; cervical fracture subluxations; or a penetrating mechanism of injury. After hospital discharge, patients who developed new neurological signs or symptoms, such as orbital bruit, delayed epistaxis, or pulsatile proptosis, required further vascular investigations during follow-up. In order to identify patients with TCVI for inclusion in the present study, we reviewed the electronic medical records of the hospital database for TBI patients from the date of injury to the last follow-up date or December 31, 2017.

In order to qualify for inclusion in the study, patients had to have developed a TCVI after a TBI. Fifty-eight patients were excluded because their medical records were inaccessible or the initial neuroimaging studies were unavailable. A total of 42 of 5178 patients with TBI developed a TCVI and had medical records and initial imaging studies available for review and were ultimately included in our analysis.

Following Bodanapally et al. ${ }^{4}$ and Biffl et al., ${ }^{3}$ TCVI was defined as intradural arterial injury from a primary force including blunt or penetrating injury and categorized into 4 groups: stenosis/occlusion; transection; pseudoaneurysm; and AVF. Cases of injuries to extracranial and extradural vessels were excluded, as were cases of cerebral infarction from secondary insults, such as a brain hypoxia.

Data extracted from the medical records included demographic characteristics, mechanism of injury, type of intracranial injury, other associated injuries, treatment, and outcome. Additionally, severity of TBI was categorized as mild, moderate, or severe according to the patient's GCS score. ${ }^{12}$

Neuroimaging studies described the types of intracranial injury, such as skull fracture, epidural hematoma, subdural hematoma, and cerebral contusion. The surgical treatments, outcome at hospital discharge, and the last follow-up were reviewed. The study was performed with permission from the Ethics Committee of the Faculty of Medicine, Songklanagarind Hospital, Prince of Songkla University.

\section{Statistical Analysis}

Patient characteristics, mechanism of injury, intracranial injury, associated injury, and TCVI characteristics were analyzed. The results are presented as proportions and mean values (with standard deviations). The test results of associations between several factors and the TCVI characteristics were analyzed by the chi-square test and Fisher's exact test. A p value $<0.05$ was considered to be statistically significant. The statistical analyses were performed with the $\mathrm{R}$ version 3.4.0 software ( $\mathrm{R}$ Foundation).

\section{Results}

We found that TCVI occurred in $0.8 \%$ of the 5178 TBI patients treated at our institution during the study period, based on a median follow-up duration of 50 days (IQR 31-120 days). Table 1 shows the clinical characteristics of the 42 patients with TCVI. More than two-thirds were male, and the mean age was $33.0 \pm 14.0$ years (range 16 72 years). The most common mechanism of injury was
TABLE 1. Clinical characteristics of 42 patients with TCVI

\begin{tabular}{|c|c|}
\hline Characteristic & Value \\
\hline \multicolumn{2}{|l|}{ Age in yrs } \\
\hline $0-19$ & $6(14.3)$ \\
\hline $20-60$ & $34(81.0)$ \\
\hline$>60$ & $2(4.8)$ \\
\hline \multicolumn{2}{|l|}{ Sex } \\
\hline Male & $32(76.2)$ \\
\hline Female & $10(23.8)$ \\
\hline \multicolumn{2}{|l|}{ Mechanism of injury } \\
\hline Motorcycle accident & $29(69.0)$ \\
\hline Car accident & $5(11.9)$ \\
\hline Fall from a height & $1(2.4)$ \\
\hline Assault & $1(2.4)$ \\
\hline Secondary blast injury & $1(2.4)$ \\
\hline Shotgun injury & $3(7.1)$ \\
\hline Object penetrating into head & $1(2.4)$ \\
\hline Pedestrian & $1(2.4)$ \\
\hline \multicolumn{2}{|l|}{ Severity } \\
\hline GCS 13-15 & $26(61.9)$ \\
\hline GCS 9-12 & $2(4.8)$ \\
\hline GCS 3-8 & $14(33.3)$ \\
\hline \multicolumn{2}{|l|}{ Signs \& symptoms } \\
\hline Proptosis & $28(66.7)$ \\
\hline Bruit & $9(21.4)$ \\
\hline Epistaxis & $4(9.5)$ \\
\hline Alteration of consciousness & $4(9.5)$ \\
\hline Cranial nerve involvement & $2(4.8)$ \\
\hline Incidental finding & $1(2.4)$ \\
\hline Mean no. of accident symptoms $\times$ days (SD) & $73.6(119.6)$ \\
\hline \multicolumn{2}{|l|}{ Pupillary reaction } \\
\hline Reactive, both pupils & $21(50.0)$ \\
\hline Unequal & $17(43.6)$ \\
\hline Non-reactive, both pupils & $4(10.4)$ \\
\hline \multicolumn{2}{|l|}{ Intracranial injury on admission date } \\
\hline Basilar skull fracture & $25(59.5)$ \\
\hline Cerebral contusion & $12(31.0)$ \\
\hline Subdural hemorrhage & $10(23.8)$ \\
\hline Subarachnoid hemorrhage & $5(11.9)$ \\
\hline Epidural hematoma & $4(9.5)$ \\
\hline Intraventricular hemorrhage & $1(2.4)$ \\
\hline \multicolumn{2}{|l|}{ Midline shift on admission date } \\
\hline$<5 \mathrm{~mm}$ & $35(83.3)$ \\
\hline$\geq 5 \mathrm{~mm}$ & $7(16.7)$ \\
\hline Basal cistern obliteration on admission date & $5(11.9)$ \\
\hline Maxillofacial injury & $14(33.3)$ \\
\hline Orbital injury & $13(31.0)$ \\
\hline
\end{tabular}

Data are $n(\%)$ unless otherwise indicated. 
TABLE 2. Injury characteristics and treatment in 42 cases of TCVI

\begin{tabular}{lc}
\hline \multicolumn{1}{c}{ Characteristic } & Value \\
\hline Type of TCVI & $35(86.4)$ \\
\hline Arteriovenous fistula & $5(11.9)$ \\
\hline Pseudoaneurysm & $1(2.4)$ \\
\hline Occlusion & $1(2.4)$ \\
\hline Transection & \\
\hline Vessel affected & $35(85.4)$ \\
\hline Internal carotid artery & $2(4.8)$ \\
\hline Posterior cerebral artery & $2(4.8)$ \\
\hline Posterior communicating artery & $1(2.4)$ \\
\hline Pericallosal artery & $1(2.4)$ \\
\hline Ophthalmic artery & $1(2.4)$ \\
\hline Middle cerebral artery & $38(90.5)$ \\
\hline Treatment & $2(4.8)$ \\
\hline Endovascular & $2(4.8)$ \\
\hline Surgical & $33.8(33.2)$ \\
\hline No definite treatment (unstable vital signs) & \\
\hline Mean duration of follow-up in mos (SD) & $3(7.1)$ \\
\hline Outcome per GoS & $1(2.4)$ \\
\hline Death & $3(7.1)$ \\
\hline Vegetative state & $15(35.6)$ \\
\hline Severe disability &
\end{tabular}

Data are $\mathrm{n}(\%)$ unless otherwise indicated.

road traffic injury (83.3\%), especially motorcycle collision $(69.0 \%)$, whereas penetrating injury occurred in only $11.9 \%$ of the cases. Most of the patients in the study population (61.9\%) had mild head injuries, and the remaining patients had moderate to severe head injuries. The common signs and symptoms of TCVI were proptosis, audible bruit, and epistaxis. Proptosis and bruit were associated with fistulas, whereas epistaxis was associated with ruptured pseudoaneurysm $(p=0.02)$. The timing of clinical symptom development related to TCVI ranged from immediate to 505 days after impact. The most common intracranial injury before TCVI was basilar skull fracture; maxillofacial and orbital injuries were found, respectively, in $14 \%$ and $13 \%$ of the 42 patients with TCVI.

According to Bodanapally et al. ${ }^{4}$ and Biffl et al., ${ }^{3}$ TCVIs are divided into 4 groups: stenosis/occlusion, pseudoaneurysm, AVF, and transection. ${ }^{28}$ Characteristics of TCVI injuries are demonstrated in Table 2 . In the present study, the two most common types of TCVI were AVF (86.4\%) and pseudoaneurysm (11.9\%). The injuries were located at the internal carotid artery (ICA; 85.4\%), the posterior cerebral artery (PCA; 4.8\%), the posterior communicating artery (PCoA; $2.4 \%)$, the pericallosal artery $(2.4 \%)$, the ophthalmic artery (2.4\%), and the middle cerebral artery (MCA; $2.4 \%$ ).

Since the most common pathology of TCVI in the present study was AVF, endovascular intervention was the
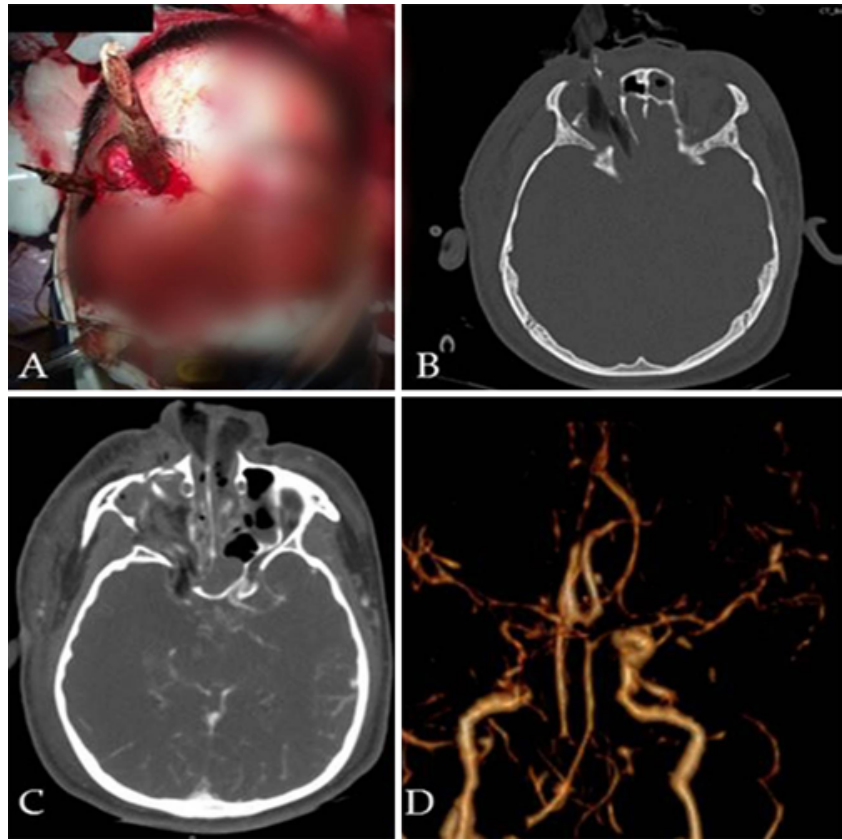

FIG. 1. Case 1: Secondary blast injury followed by right ICA occlusion. A: Photograph showing penetration of wooden fragments into the right orbit at both medial and lateral sites. Blurring has been applied for privacy. B: Axial CT bone-window image showing wooden fragments in the right orbit. C: Axial CT angiogram showing the tip of a fragment through the orbital apex and compressing the right ICA. D: 3D reconstruction image showing reperfusion into the right ICA via the circle of Willis.

major management. However, surgical management could play a role in pseudoaneurysms, depending on the location and architecture of the aneurysm. Outcomes of TCVI varied in severity and included good recovery $(35.7 \%)$, moderate disability $(47.6 \%)$, severe disability $(7.1 \%)$, vegetative state $(2.4 \%)$, and death (7.1\%), according to the Glasgow Outcome Scale (GOS) score.

\section{Illustrative Cases}

\section{Case 1: ICA Occlusion From Secondary Blast Injury}

A 26-year-old soldier was injured by an explosion while on duty. Wooden fragments penetrated the right orbit (Fig. 1A). CT scans of the patient's head demonstrated that the tip of one of these fragments had penetrated to the anterior clinoid process, causing a right ICA occlusion (Fig. $1 \mathrm{~B}$ and $\mathrm{C}$ ). The treatment plan was to restore blood flow to the supraclinoid part of the ICA, MCA, and PCA via the patent circle of Willis (Fig. 1D). Unfortunately, the patient developed unstable vital signs due to hemorrhage (with bleeding from the nose and ear) after the initial investigations. Although resuscitation and massive blood transfusion were performed, the patient had persistent hypotension and died 1 day later.

\section{Case 2: MCA Transection With Contrast Extravasation}

A 34-year-old male patient sustained a shotgun injury to the head. At the scene, he developed cardiac arrest and received cardiopulmonary resuscitation. After resuscita- 

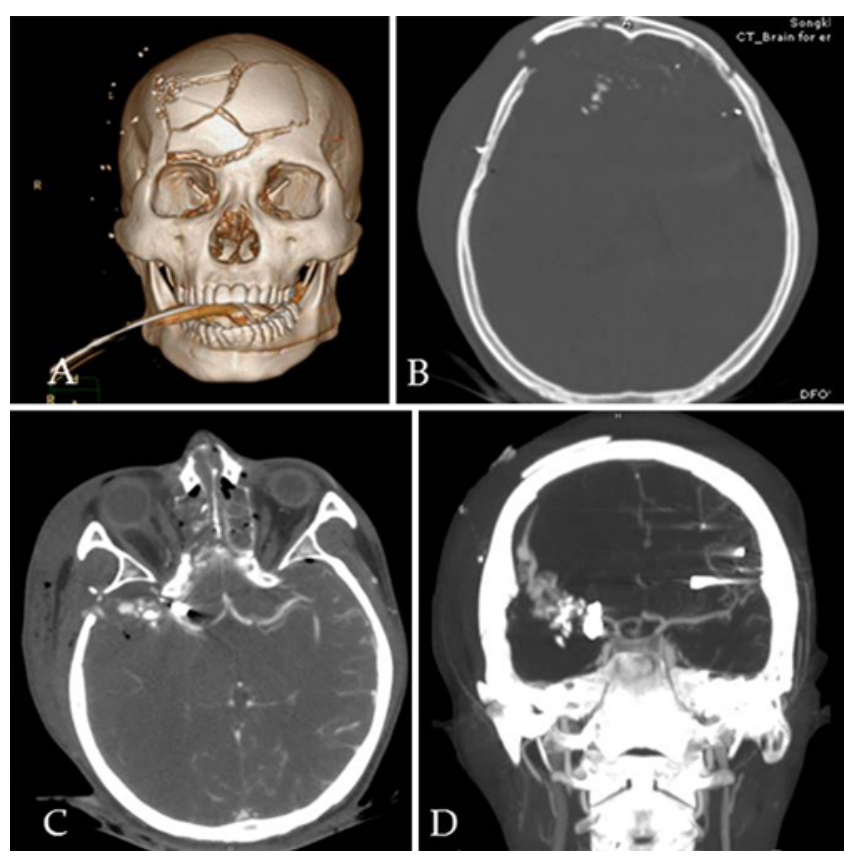

FIG. 2. Case 2: Shotgun injury followed by right MCA transection with contrast extravasation. $\mathrm{A}: 3 \mathrm{D}$ reconstruction of the skull showing a mosaic fracture of the bifrontal bone. B: Axial CT head bone-window image showing the bifrontal bone fracture and multiple pellets. C: Axial CTA head showing metallic pellets through the right MCA. D: Coronal CTA head showing contrast extravasation via transected MCA on the right side. tion, his vital signs returned after arrival at the hospital but he had a GCS score of 3 with fixed dilated pupils. The head CT showed bilateral frontal bone fractures with retained multiple shotgun pellets in both frontal lobes (Fig. 2A and B). CTA showed that pellets had penetrated the right MCA, and contrast extravasation was observed (Fig. $2 \mathrm{C}$ and $\mathrm{D}$ ). The patient developed hypotension and a second cardiac arrest. Unfortunately, this time he was unresponsive to cardiopulmonary resuscitation and died.

\section{Case 3: Right PCA Pseudoaneurysm}

A 17-year-old male patient was injured in a motorcycle collision. Upon initial examination, he had a GCS score of 15 and was bleeding from the right ear. A CT scan demonstrated subarachnoid hemorrhage at the suprasellar, interpeduncular, and ambient cistern with basilar skull fracture (Fig. 3A and B). He was admitted for close observation, but developed deterioration of consciousness within 2 hours after admission. A repeat CT brain revealed new right temporal contusion and subarachnoid hemorrhage with 6.1-mm thickness behind the dorsum sellae (Fig. 3C). Decompressive craniectomy was performed. The GOS score at hospital discharge was 2 (vegetative state). One month after discharge, the patient's surgical wound was tense, and a follow-up CT of the brain showed hydrocephalus and an enhanced oval mass placed in the third ventricle (Fig. 3D). The 3D reconstruction image of the intracranial vessels showed a bilobulated aneurysm rising from the right PCA that was believed to have developed as a result of the vessel
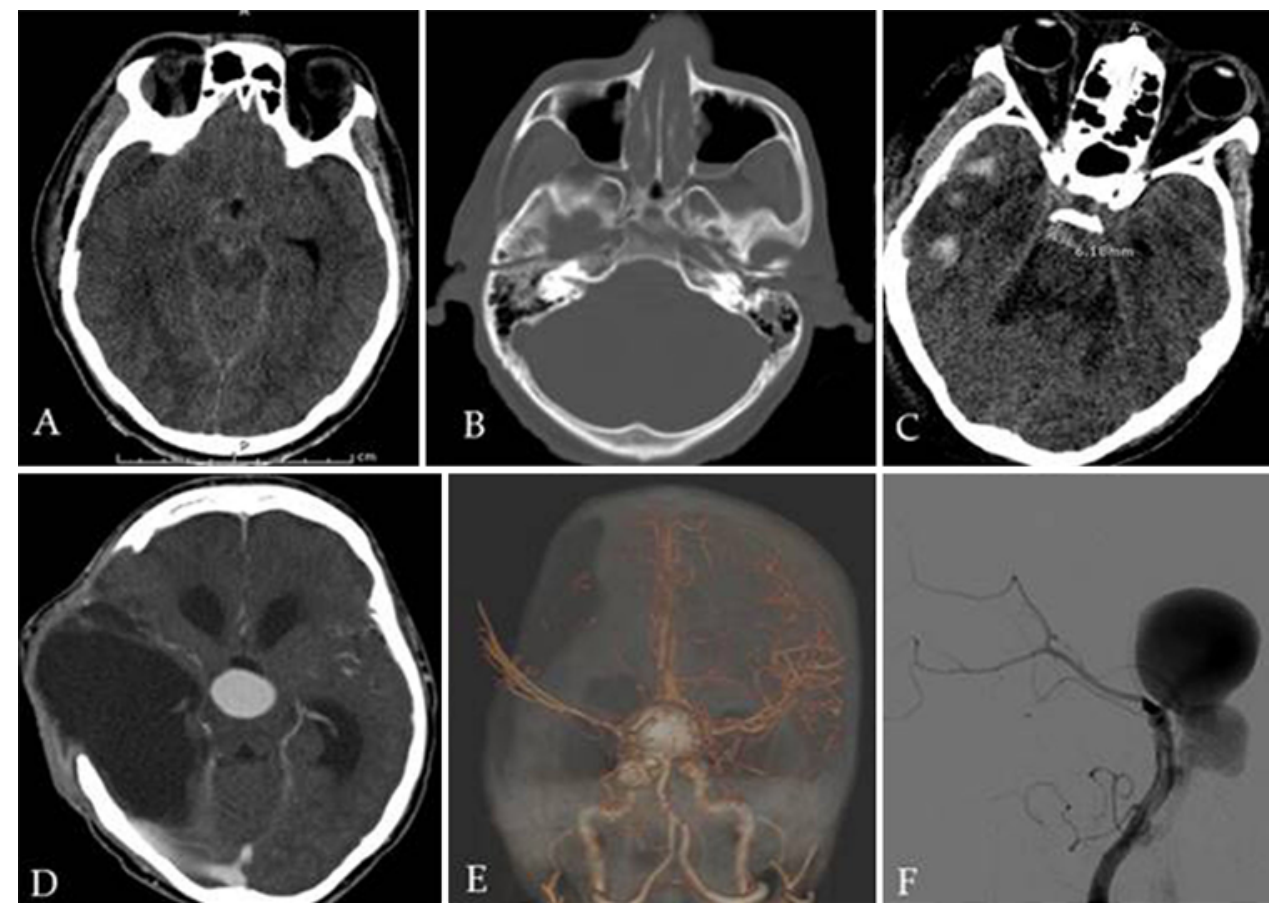

FIG. 3. Case 3: TBI from motorcycle collision followed by right PCA aneurysm. A: Axial CT showing diffuse subarachnoid hemorrhage at the basal cistern. B: Axial CT bone-window image showing right petrous bone fracture. C: Repeat CT showing new right temporal contusion with subarachnoid hemorrhage at the prepontine cistern. D: Follow-up CT showing hydrocephalus and an enhanced oval mass in the third ventricle. $E$ and F: 3D reconstruction of CT angiogram ( $E$, coronal view) of intracranial vessels and digital subtraction angiogram ( $F$, sagittal view) showing a bilobulated right PCA aneurysm. 
impacting the dorsum sellae (Fig. 3E and F). Endovascular coiling was successfully performed. As of the most recent follow-up, the patient remained in a vegetative state.

\section{Discussion}

Mechanical force loading on a vessel generates TCVI via the front of the head. Therefore, orbital, maxillofacial, and basilar skull fractures are common contact injuries. Characteristics of TCVI have been described in various types. In 1999, Biffl et al. ${ }^{3}$ reported a grading scale for blunt carotid arterial injury, distinguishing 5 injury grades: grade I, "luminal irregularity or dissection with $<25 \%$ luminal narrowing"; grade II, dissection or intramural hematoma with $\geq 25 \%$ luminal narrowing, intraluminal thrombus, or raised intimal flap"; grade III, pseudoaneurysm; grade IV, occlusion; and grade V, "transection with free extravasation." Bodanapally et al., ${ }^{4}$ in their 2015 article on arterial injuries after penetrating brain injury, described 4 types of intracranial arterial injury: traumatic intracranial aneurysm, dissection, occlusion, and carotidcavernous fistulas. In this current study, we stratified the types of TCVI into 4 groups: stenosis/occlusion, pseudoaneurysm, AVF, and transection..$^{28}$

Cerebral angiography is the standard tool for detecting TCVI; however, this method is invasive and sometimes inappropriate in an acute setting. CTA was reported to be the appropriate initial diagnostic tool in an emergency setting because it is less time-consuming and less invasive. ${ }^{10,19}$ The sensitivity of CTA to detect intracerebral vascular injuries is $72.7 \%$ (95\% CI $49.8 \%-89.3 \%),{ }^{5}$ while the sensitivity of a CTA to detect carotid artery injury of the neck ranges from $98 \%$ to $100 \%$. CTA has lower sensitivity to detect a TCVI because the intracranial arteries are noticeably smaller in caliber, and beam-hardening metallic artifacts impair resolution of intracranial vessels. 2,10,19,20,24 However, screening for TCVI with CTA is accepted practice in traumatic patients before confirming a diagnosis from cerebral angiography.

TCVIs may be caused by both direct and indirect forces on a vascular surface and have various manifestations, which depend on the morphology of the lesion. Synchronized injuries of the artery and the adjacent vein may lead to a pathophysiological formation of an AVF. ${ }^{8}$ The pathogenesis of an occlusion is classified into 4 causes: embolism from the ICA, vasospasm, thrombus formation, and dissection..$^{18}$ Additionally, a force that sharply cuts vessels results in complete transection with contrast extravasation. Pseudoaneurysms occur from a shearing force that deforms the wall of a vessel. ${ }^{17}$ A PCA aneurysm can occur when the vessel accelerates to the sharp edge of the tentorium cerebelli, dorsum sellae, or clivus. ${ }^{17,32}$ Moreover, if the pericallosal artery is located near the falx cerebri in the coronal plane, such an incident may lead to the formation of a pericallosal artery aneurysm. ${ }^{21,23}$ Additionally, a direct force from a penetrating injury such as a gunshot wound or secondary blast injury can strain the arterial wall and allow blood to leak through the wall and then be contained by the adventitia or surrounding perivascular soft tissue. ${ }^{17}$

The mortality in the present study was $7.1 \%$ of overall TCVI cases. However, Onda et al. reported a TCVI-related death rate of $14.3 \% .{ }^{22}$ Early detection and treatment are essential for optimal outcome. The two common treatment options for TCVI are surgery and endovascular coil embolization. Endovascular intervention is feasible in cases of AVF, $, 25,30$ and treatments of pseudoaneurysm have been discussed elsewhere. ${ }^{14,25,29}$ Based on expert opinion, the type of TCVI and anatomical considerations, such as size, location, and morphological features, determine which treatment is most appropriate for the individual patient. The treatment approach must be considered on a case-bycase basis because atypical locations and variable shapes are often encountered. Surgical clipping, repair, trapping, or excision and bypass of an aneurysm are considered to be surgical treatment options, whereas endovascular interventions such as coiling and endovascular occlusion are alternative treatments for distal branch or fusiform aneurysms. ${ }^{13,15,29}$

In traumatic vascular occlusion or stenosis management, intravascular volume expansion/hemodilution therapies are mentioned in the literature, but the outcomes are poor due to secondary cerebral infarction..$^{26,31}$ In our first illustrative case, a surgical approach to remove the wooden foreign bodies with proximal control preparation was the plan. Unfortunately, the patient's clinical status was unstable and he died. Early diagnosis and treatment of transection are crucial. CTA is a rapid and effective diagnostic test. The next step is either an endovascular approach or surgical repair. ${ }^{1}$ Endovascular interventions may be preferable in cases of transection because surgical treatment is time-consuming. $6.7,22$

We recognize that this study is limited by its retrospective design and that the number of TCVI cases might be underdetected because our institution did not routinely perform either cerebral angiography or CTA of the brain in all TBI patients. However, vascular imaging of all patients with TBI would not represent a typical real-world situation. In patients considered to be at high risk for vascular injuries, such as those with penetrating injury, severe head injury, or basilar skull fracture, $, 15,17,28$ cerebral angiography or CTA should be performed.

\section{Conclusions}

We propose types and characteristics of TCVIs. A TCVI is an uncommon complication of TBI, but it can be life-threatening. Early detection allows for prompt treatment and should help improve outcomes.

\section{Acknowledgments}

We would like to offer our special thanks to Dr. Kittipong Riabroi and Dr. Rujimas Khumtong for their advice about cerebral angiogram information. Furthermore, we thank Dr. Osaree Akaraborworn for the funding from the Targeted Research Grants, Faculty of Medicine, Prince of Songkla University.

\section{References}

1. Atar E, Griton I, Bachar GN, Bartal G, Kluger Y, Belenky A: Embolization of transected vertebral arteries in unstable trauma patients. Emerg Radiol 11:291-294, 2005

2. Baerlocher MO, Zakrison TL, Tien H, Aviv RI, Symons SP: Traumatic cervical vertebral artery transection associated 
with a dural tear leading to subarachnoid extravasation. Eur J Trauma Emerg Surg 35:67-70, 2009

3. Biffl WL, Moore EE, Offner PJ, Brega KE, Franciose RJ, Burch JM: Blunt carotid arterial injuries: implications of a new grading scale. J Trauma 47:845-853, 1999

4. Bodanapally UK, Saksobhavivat N, Shanmuganathan K, Aarabi B, Roy AK: Arterial injuries after penetrating brain injury in civilians: risk factors on admission head computed tomography. J Neurosurg 122:219-226, 2015

5. Bodanapally UK, Shanmuganathan K, Boscak AR, Jaffray PM, Van der Byl G, Roy AK, et al: Vascular complications of penetrating brain injury: comparison of helical CT angiography and conventional angiography. J Neurosurg 121:12751283,2014

6. Cohen JE, Rajz G, Itshayek E, Umansky F, Gomori JM: Endovascular management of exsanguinating vertebral artery transection. Surg Neurol 64:331-334, 2005

7. de Castro SM, Christiaans SC, van den Berg R, Schep NW: Minimal invasive management of traumatic transection of the vertebral artery. Springerplus 3:206, 2014

8. Fattahi TT, Brandt MT, Jenkins WS, Steinberg B: Traumatic carotid-cavernous fistula: pathophysiology and treatment. J Craniofac Surg 14:240-246, 2003

9. Gemmete JJ, Chaudhary N, Pandey A, Ansari S: Treatment of carotid cavernous fistulas. Curr Treat Options Neurol 12:43-53, 2010

10. Inaba K, Munera F, McKenney M, Rivas L, de Moya M, Bahouth $\mathrm{H}$, et al: Prospective evaluation of screening multislice helical computed tomographic angiography in the initial evaluation of penetrating neck injuries. J Trauma 61:144149,2006

11. Ishibashi A, Kubota Y, Yokokura Y, Soejima Y, Hiratsuka T: Traumatic occlusion of the anterior cerebral artery-case report. Neurol Med Chir (Tokyo) 35:882-885, 1995

12. Jennett B, Bond M: Assessment of outcome after severe brain damage. Lancet 1:480-484, 1975

13. Jung SH, Kim SH, Kim TS, Joo SP: Surgical treatment of traumatic intracranial aneurysms: experiences at a single center over 30 years. World Neurosurg 98:243-250, 2017

14. Kobata H: Diagnosis and treatment of traumatic cerebrovascular injury: pitfalls in the management of neurotrauma. Neurol Med Chir (Tokyo) 57:410-417, 2017

15. Larson PS, Reisner A, Morassutti DJ, Abdulhadi B, Harpring JE: Traumatic intracranial aneurysms. Neurosurg Focus 8(1):e4, 2000

16. Martin S, Teo M, Bhattacharya J, Alakandy L: Caroticocavernous fistula: an educational case. Int J Surg Case Rep 4:858-860, 2013

17. Miley JT, Rodriguez GJ, Qureshi AI: Traumatic intracranial aneurysm formation following closed head injury. J Vasc Interv Neurol 1:79-82, 2008

18. Miyata M, Yamasaki S, Hirayama A, Tamaki N: [Traumatic middle cerebral artery occlusion.] No Shinkei Geka 22:253257, 1994 (Japanese)

19. Múnera F, Soto JA, Palacio D, Velez SM, Medina E: Diagnosis of arterial injuries caused by penetrating trauma to the neck: comparison of helical CT angiography and conventional angiography. Radiology 216:356-362, 2000

20. Múnera F, Soto JA, Palacio DM, Castañeda J, Morales C, Sanabria A, et al: Penetrating neck injuries: helical CT angiography for initial evaluation. Radiology 224:366-372, 2002

21. Nakstad P, Nornes H, Hauge HN: Traumatic aneurysms of the pericallosal arteries. Neuroradiology 28:335-338, 1986
22. Onda H, Fuse A, Yamaguchi M, Igarashi Y, Watanabe A, Suzuki G, et al: Traumatic cerebrovascular injury following severe head injury: proper diagnostic timetable and examination methods. Neurol Med Chir (Tokyo) 53:573-579, 2013

23. Quintana F, Diez C, Gutierrez A, Diez ML, Austin O, Vazquez A: Traumatic aneurysm of the basilar artery. AJNR Am J Neuroradiol 17:283-285, 1996

24. Steenburg SD, Sliker CW, Shanmuganathan K, Siegel EL: Imaging evaluation of penetrating neck injuries. Radiographics 30:869-886, 2010

25. Sui M, Mei Q, Sun K: Surgical treatment achieves better outcome in severe traumatic pericallosal aneurysm: case report and literature review. Int J Clin Exp Med 8:1598-1603, 2015

26. Suwanwela C, Suwanwela N: Intracranial arterial narrowing and spasm in acute head injury. J Neurosurg 36:314-323, 1972

27. Tunthanathip T, Khocharoen K, Phuenpathom N: Blastinduced traumatic brain injury: the experience from a level I trauma center in southern Thailand. Neurosurg Focus 45(6):E7, 2018

28. Tunthanathip T, Phuenpathom N, Saehaeng S, Oearsakul T, Sakarunchai I, Kaewborisutsakul A: Traumatic cerebrovascular injury: prevalence and risk factors. Am J Emerg Med [epub ahead of print], 2019

29. Van Rooij WJ, Van Rooij SB: Endovascular treatment of traumatic pericallosal artery aneurysms. A case report. Interv Neuroradiol 19:56-59, 2013

30. Wu Z, Zhang Y, Wang C, Yang X, Li Y: Treatment of traumatic carotid-cavernous fistula. Interv Neuroradiol 6:277289,2000

31. Yamada K, Harada M, Hasegawa S, Ushio Y: Delayed posttraumatic middle cerebral artery vasospasm demonstrated by magnetic resonance angiography: case report. Neurosurgery 43:153-156, 1998

32. Zhang C, Chen H, Bai R: Traumatic aneurysm on the posterior cerebral artery following blunt trauma in a 14-year-old girl: case report. Neuropediatrics 42:204-206, 2011

\section{Disclosures}

The authors report no conflict of interest concerning the materials or methods used in this study or the findings specified in this paper.

\section{Author Contributions}

Conception and design: Tunthanathip, Kaewborisutsakul. Acquisition of data: Tunthanathip. Analysis and interpretation of data: Tunthanathip. Drafting the article: Tunthanathip, Oearsakul, Sakarunchai, Kaewborisutsakul. Critically revising the article: Tunthanathip. Reviewed submitted version of manuscript: all authors. Approved the final version of the manuscript on behalf of all authors: Tunthanathip. Statistical analysis: Tunthanathip. Administrative/technical/material support: Tunthanathip. Study supervision: Phuenpathom, Sae-Heng, Oearsakul.

\section{Correspondence}

Thara Tunthanathip: Prince of Songkla University, Hat Yai, Songkhla, Thailand. tsus4@hotmail.com. 